\title{
Inertial KM-type extragradient scheme for solving a variational inequality and a hierarchical fixed point problems
}

\author{
Ghada AlNemer ${ }^{1}$, Rehan $\mathrm{Ali}^{2}$ and K.R. Kazmi $\mathrm{i}^{3,4^{*}}$
}

\section{"Correspondence:}

krkazmi@gmail.com

${ }^{3}$ Deparment of Mathematics,

Faculty of Science and Arts-Rabigh King Abdulaziz University, P.O. Box 344, Rabigh 21911, Saudi Arabia

${ }^{4}$ Department of Mathematics, Aligarh Muslim University, 202002

Aligarh, India

Full list of author information is available at the end of the article

\begin{abstract}
We propose an inertial KM-type extragradient scheme to approximate a common solution of a variational inequality problem and a hierarchical fixed point problem for nonexpansive mappings. This scheme generalizes and unifies a number of known iterative schemes. Furthermore, we discuss the weak convergence for the proposed scheme. We also discuss an example to illustrate the main theorem.
\end{abstract}

MSC: 47H10;49J35; 90C47

Keywords: Hierarchical fixed point problem; Variational inequality; Inertial KM-type scheme; Nonexpansive mapping

\section{Introduction}

Let $\mathcal{C}$ be a nonempty convex and closed set in a real Hilbert space $\mathcal{H}$ and $\langle\cdot, \cdot\rangle$ and $\|\cdot\|$ denote the inner product and induced norm on $\mathcal{H}$. A mapping $U: \mathcal{C} \rightarrow \mathcal{C}$ is said to be nonexpansive if $\|U u-U v\| \leq\|u-v\|, \forall u, v \in \mathcal{C}$. Note that if $\mathrm{F}(U):=\{u \in \mathcal{C}: U u=u\} \neq \emptyset$ then set $\mathrm{F}(U)$ is convex and closed. Let $\mathrm{F}(U) \neq \emptyset$. The subdifferential of a proper function $g: \mathcal{H} \rightarrow(-\infty,+\infty]$ is the set-valued operator $\partial g: \mathcal{H} \rightarrow 2^{\mathcal{H}}$ defined by $\partial g(u)=\{w \in \mathcal{H}:$ $\langle y-u, w\rangle+g(u) \leq g(y), \forall y \in \mathcal{H}\}$. Let $u \in \mathcal{H}$. Then $g$ is subdifferential at $u$ if $\partial g(u) \neq \emptyset$. The indicator function $\psi_{\mathcal{C}}: \mathcal{H} \rightarrow(-\infty,+\infty]$ is given by

$$
\partial \psi_{\mathcal{C}}(u)= \begin{cases}0, & u \in \mathcal{C}, \\ \infty, & \text { otherwise }\end{cases}
$$

Note that $\psi_{\mathcal{C}}$ is a convex function when $\mathcal{C}$ is a convex set.

In 2006, Moudafi et al. [1] discussed the convergence of a scheme for the following hierarchical fixed point problem (in short, H-FPP): Find $\bar{u} \in \mathrm{F}(U)$ such that

$$
\langle\bar{u}-V \bar{u}, \bar{u}-u\rangle \leq 0, \quad \forall u \in \mathrm{F}(U),
$$

where the mappings $U, V: \mathcal{C} \rightarrow \mathcal{C}$ are nonexpansive. Let $\Phi$ denote the set of solutions of H-FPP(1.1). If $\bar{u} \in \mathrm{F}(U)$ then $(1.1) \Leftrightarrow\langle-(I-V) \bar{u}, u-\bar{u}\rangle+\psi_{\mathrm{F}(U)}(\bar{u}) \leq \psi_{\mathrm{F}(U)}(u) \Leftrightarrow-(I-V) \bar{u} \in$

(c) The Author(s) 2021. This article is licensed under a Creative Commons Attribution 4.0 International License, which permits use, sharing, adaptation, distribution and reproduction in any medium or format, as long as you give appropriate credit to the original author(s) and the source, provide a link to the Creative Commons licence, and indicate if changes were made. The images or other third party material in this article are included in the article's Creative Commons licence, unless indicated otherwise in a credit line to the material. If material is not included in the article's Creative Commons licence and your intended use is not permitted by statutory regulation or exceeds the permitted use, you will need to obtain permission directly from the copyright holder. To view a copy of this licence, visit http://creativecommons.org/licenses/by/4.0/ 
$\partial \psi_{\mathrm{F}(U)}(\bar{u})$. Hence H-FPP(1.1) is equivalent to the variational inclusion: Find $\bar{u} \in \mathrm{F}(U)$ such that

$$
0 \in(I-V) \bar{u}+N_{\mathrm{F}(U)}(\bar{u}),
$$

where the mapping $I$ is identity on $\mathcal{C}$ and $N_{\mathrm{F}(U)}(\bar{u})$ denotes the normal cone to $\mathrm{F}(U)$ at $\bar{u}$ given by

$$
N_{\mathrm{F}(U)}(\bar{u})=\partial \psi_{\mathrm{F}(U)}(\bar{u})= \begin{cases}\{w \in \mathcal{H}:\langle u-\bar{u}, w\rangle \leq 0, \forall u \in \mathrm{F}(U)\}, & \text { if } \bar{u} \in \mathrm{F}(U), \\ \emptyset, & \text { otherwise. }\end{cases}
$$

If we set $V=I$, then $\Phi$ is just $\mathrm{F}(U)$. Furthermore, we mention that H-FPP(1.1) is worth to study because it includes as special cases, the important problems such as the variational inequality on fixed point sets and hierarchical minimization problems; see Moudafi [2].

In 2007, Moudafi [2] proposed the following Krasnoselski-Mann (KM)-type scheme for solving H-FPP(1.1): For given $u_{0} \in \mathcal{C}$,

$$
u_{k+1}=\left(1-\alpha_{k}\right) u_{k}+\alpha_{k}\left(\sigma_{k} V u_{k}+\left(1-\sigma_{n}\right) U u_{k}\right), \quad \forall n \geq 0,
$$

where $\left\{\alpha_{k}\right\} \subset(0,1)$ and $\left\{\sigma_{k}\right\} \subset(0,1)$. For further work related to scheme (1.3), see for example [1,3-7].

In 2008, Mainge [8] introduced an inertial version of KM-type scheme by unifying the KM-type scheme and the inertial extrapolation, for approximating a fixed point of nonexpansive mappings and discussed the weak convergence. Recently, Bot et al. [9] derived some the convergence results of the following inertial KM-type scheme to approximate a fixed point of nonexpansive mapping $U$ on $\mathcal{H}$ which generalize the results of Mainge [8]:

$$
\left.\begin{array}{l}
t_{k}=u_{k}+\eta_{k}\left(u_{k}-u_{k-1}\right) \\
u_{k+1}=\left(1-\alpha_{k}\right) t_{k}+\alpha_{k} U t_{k}
\end{array}\right\}
$$

for each $k \geq 1$, where $\eta_{k}$ is a damping-type term and $\alpha_{k}$ is a relaxation factor. Recently, the interest of studying inertial type algorithms has been increased due to their fast convergence. For further study of scheme (1.4) and its generalizations; see for example [10-13].

On the other hand, we consider the classical variational inequality (in short, VI): Find $\bar{u} \in \mathcal{C}$ such that

$$
\langle h(\bar{u}), v-\bar{u}\rangle \geq 0, \quad \forall v \in \mathcal{C},
$$

introduced in [14] where $h: \mathcal{H} \rightarrow \mathcal{H}$. The set of solutions of $\mathrm{VI}(1.5)$ is denoted by $\mathrm{Sol}(\mathrm{VI}(1.5))$. Note that the projected gradient scheme for solving $\mathrm{VI}(1.5)$ is

$$
u_{k+1}=\mathcal{P}_{\mathcal{C}}\left(u_{k}-\mu h\left(u_{k}\right)\right) \text {, }
$$

where $\mu>0$ and $\mathcal{P}_{\mathcal{C}}$ is the metric projection onto $\mathcal{C}$. In order to converge, this scheme requires the restrictive condition that $h$ is inverse strongly (or strongly) monotone. To 
overcome this difficulty, Korpelevich [15] proposed an extragradient iterative scheme by

$$
\left.\begin{array}{l}
v_{k}=\mathcal{P}_{\mathcal{C}}\left(u_{k}-\mu h\left(u_{k}\right)\right) \\
u_{k+1}=\mathcal{P}_{\mathcal{C}}\left(u_{k}-\mu h\left(v_{k}\right)\right)
\end{array}\right\}
$$

where $\mu \in\left(0, \frac{1}{L}\right)$, where $L>0$ is Lipschitz constant of $h$. Since then many researchers improved scheme (1.7) in various directions; see, e.g. [16-24] and the references therein. Note that the calculation of two projections onto $\mathcal{C}$ might affect the efficiency of such scheme. Therefore, Dong et al. [25] proposed the following inertial KM-type extragradient scheme for VI(1.5):

$$
\left.\begin{array}{l}
t_{k}=u_{k}+\eta_{k}\left(u_{k}-u_{k-1}\right), \\
v_{k}=\mathcal{P}_{\mathcal{C}}\left(t_{k}-\mu h\left(t_{k}\right)\right) \\
u_{k+1}=\left(1-\alpha_{k}\right) t_{k}+\alpha_{k} \mathcal{P}_{\mathcal{C}}\left(t_{k}-\mu h\left(v_{k}\right)\right),
\end{array}\right\}
$$

where $\left\{\eta_{k}\right\} \subset[0, \eta], \forall k$ is nondecreasing with $\eta_{1}=0$ and $0 \leq \eta_{k} \leq \eta<1$, for every $k \geq 1$ such that

$$
\delta>\frac{\eta\left[(1+\mu L)^{2} \eta(1+\eta)+\left(1-\mu^{2} L^{2}\right) \eta \sigma+\sigma(1+\mu L)^{2}\right]}{1-\mu^{2} L^{2}}
$$

and

$$
0<\alpha \leq \alpha_{k} \leq \frac{\delta\left(1-\mu^{2} L^{2}\right)-\eta\left[(1+\mu L)^{2} \eta(1+\eta)+\left(1-\mu^{2} L^{2}\right) \eta \sigma+\sigma(1+\mu L)^{2}\right]}{\delta\left[(1+\mu L)^{2} \eta(1+\eta)+\left(1-\mu^{2} L^{2}\right) \eta \sigma+\sigma(1+\mu L)^{2}\right]},
$$

where $\alpha, \sigma, \delta>0$.

They proved the weak convergence theorem for scheme (1.8).

In this paper, we propose an inertial version of KM-type extragradient scheme by combining iterative schemes (1.3) and (1.8) to approximate a common solution of H-FPP(1.1) and VI(1.5). We prove a weak convergence theorem for the proposed scheme. Furthermore, we discuss an example to illustrate the main theorem. The theorems of the paper unify and generalize previously known corresponding theorems; see for example $[2,8,9,25-27]$.

\section{Preliminaries}

We give some definitions and results of convex and nonlinear analysis, which will be used in the proof of the weak convergence theorem.

A mapping $\mathcal{P}_{\mathcal{C}}$ is called the metric projection of $\mathcal{H}$ onto $\mathcal{C}$ if for every point $u \in \mathcal{H}$, there exists a unique point in $\mathcal{C}$ denoted by $\mathcal{P}_{\mathcal{C}} u$ such that

$$
\left\|u-\mathcal{P}_{\mathcal{C}} u\right\| \leq\|u-v\|, \quad \forall v \in \mathcal{C} .
$$

Note that $\mathcal{P}_{\mathcal{C}}$ is nonexpansive and satisfies

$$
\left\langle u-v, \mathcal{P}_{\mathcal{C}} u-\mathcal{P}_{\mathcal{C}} v\right\rangle \geq\left\|\mathcal{P}_{\mathcal{C}} u-\mathcal{P}_{\mathcal{C}} v\right\|^{2}, \quad \forall u \in \mathcal{H}
$$


Moreover, $\mathcal{P}_{\mathcal{C}} u$ is characterized by the fact $\mathcal{P}_{\mathcal{C}} u \in \mathcal{C}$ and

$$
\left\langle u-\mathcal{P}_{\mathcal{C}} u, v-\mathcal{P}_{\mathcal{C}} u\right\rangle \leq 0, \quad \forall v \in \mathcal{C},
$$

which implies that

$$
\|u-v\|^{2} \geq\left\|u-\mathcal{P}_{\mathcal{C}} u\right\|^{2}+\left\|v-\mathcal{P}_{\mathcal{C}} u\right\|^{2}, \quad \forall u \in \mathcal{H}, v \in \mathcal{C} .
$$

Definition 2.1 A mapping $h: \mathcal{H} \rightarrow \mathcal{H}$ is called:

(i) monotone, if for all $u, v \in \mathcal{H}$, we have

$$
\langle h u-h v, u-v\rangle \geq 0
$$

(ii) L-Lipschitz continuous, if there exists a constant $L>0$ such that, for all $u, v \in \mathcal{H}$, we have

$$
\|h u-h v\| \leq L\|u-v\| .
$$

Lemma 2.1 If a mapping $U$ is nonexpansive on $\mathcal{H}$ then $I-U$ is maximal monotone [28] and demiclosed [29] on $\mathcal{H}$.

Lemma 2.2 ([30]) Let $\left\{\psi_{k}\right\},\left\{\delta_{k}\right\}$ and $\left\{\eta_{k}\right\}$ be the sequences in $[0, \infty)$ such that $\psi_{k+1} \leq \psi_{k}+$ $\eta_{k}\left(\psi_{k}-\psi_{k-1}\right)+\gamma_{k}, \forall k \geq 1, \sum_{k=1}^{\infty} \gamma_{k}<+\infty$ and there is a number $\eta$ with $0 \leq \eta_{k} \leq \eta<1$, $\forall k \geq 1$. Then the following hold:

(a) $\sum_{k=1}^{\infty}\left[\psi_{k}-\psi_{k-1}\right]_{+}<+\infty$, where $[r]_{+}:=\max \{r, 0\}$;

(b) there is a $\psi^{*} \in[0, \infty)$ such that $\lim _{k \rightarrow \infty} \psi_{k}=\psi^{*}$.

Lemma 2.3 ([31]) Let $\mathcal{C}$ be a nonempty subset of $\mathcal{H}$ and the sequence $\left\{u_{k}\right\}$ in $\mathcal{H}$ satisfy the conditions:

(a) $\lim _{k \rightarrow \infty}\left\|u_{k}-u\right\|$ exists for every $u \in \mathcal{C}$;

(b) any weak cluster point of $\left\{u_{k}\right\}$ is in $\mathcal{C}$.

Then $\left\{u_{k}\right\}$ is weak convergent to a point in $\mathcal{C}$.

\section{Weak convergence theorem}

We propose the following inertial KM-type extragradient scheme for solving H-FPP(1.1) and $\mathrm{VI}(1.5)$.

Scheme Choose initial values $u_{0}, u_{1} \in \mathcal{H}$ arbitrarily. The sequence $\left\{u_{k}\right\}$ be generated by the scheme:

$$
\left.\begin{array}{l}
t_{k}=u_{k}+\eta_{k}\left(u_{k}-u_{k-1}\right), \\
v_{k}=\mathcal{P}_{\mathcal{C}}\left(t_{k}-\mu h\left(t_{k}\right)\right), \\
w_{k}=\mathcal{P}_{\mathcal{C}}\left(t_{k}-\mu h\left(v_{k}\right)\right), \\
u_{k+1}=\left(1-\alpha_{k}\right) t_{k}+\alpha_{n}\left(\sigma_{k} V w_{k}+\left(1-\sigma_{n}\right) U w_{k}\right),
\end{array}\right\}
$$


where $\left\{\eta_{k}\right\} \subset[0, \eta], \forall k$, is nondecreasing with $\eta_{1}=0$ and $0 \leq \eta_{k} \leq \eta<1,\left\{\sigma_{k}\right\} \subseteq[c, d]$, $c, d \in(0,1), \mu \in\left(0, \frac{1}{L}\right), L>0$ and $\left\{\alpha_{k}\right\}$ is a real sequence with conditions:

$$
\delta>\frac{\eta^{2}(1+\eta)+\eta \sigma}{1-\eta^{2}} \quad \text { and } \quad 0<\alpha \leq \alpha_{k} \leq \frac{\delta-\eta[\eta(1+\eta)+\eta \delta+\sigma]}{\delta[1+\eta(1+\eta)+\eta \delta+\sigma]} \text {, where } \alpha, \sigma, \delta>0
$$

Now, we discuss the weak convergence for scheme (3.1).

Theorem 3.1 Let $\mathcal{H}$ be a real Hilbert space and $\mathcal{C} \subset \mathcal{H}$ be a nonempty, convex and closed set; let the mappings $U, V: \mathcal{C} \rightarrow \mathcal{C}$ be nonexpansive and $h: \mathcal{H} \rightarrow \mathcal{H}$ be L-Lipschitz continuous and monotone. Assume that $\Gamma=\operatorname{Sol}(\mathrm{VI}(1.5)) \cap \Phi \cap \mathrm{F}(V) \neq \emptyset$. Let the sequence $\left\{u_{k}\right\}$ be defined by scheme (3.1). Then the following results hold:

(a) $\sum_{k=1}^{\infty}\left\|u_{k+1}-u_{k}\right\|^{2}<+\infty$;

(b) the sequence $\left\{u_{k}\right\}$ converges weakly to $\bar{u} \in \Gamma$.

Proof (a). Let for any $q \in \Gamma$. Since $h$ is $L$-Lipschitz continuous and monotone then we can easily get

$$
\left\|w_{k}-q\right\|^{2} \leq\left\|t_{k}-q\right\|^{2}-\left(1-\mu^{2} L^{2}\right)\left\|t_{k}-v_{k}\right\|^{2}
$$

see [3]. From the nonexpansivity of $\mathcal{P}_{\mathcal{C}}$ and Lipschitz continuity of $h$, it follows that

$$
\begin{aligned}
\left\|v_{k}-w_{k}\right\|=\left\|\mathcal{P}_{\mathcal{C}}\left(t_{k}-\mu h\left(t_{k}\right)\right)-\mathcal{P}_{\mathcal{C}}\left(t_{k}-\mu h\left(v_{k}\right)\right)\right\| & \leq \mu\left\|h\left(t_{k}\right)-h\left(v_{k}\right)\right\| \\
& \leq \mu L\left\|t_{k}-v_{k}\right\|,
\end{aligned}
$$

which yields

$$
\left\|t_{k}-w_{k}\right\| \leq\left\|t_{k}-v_{k}\right\|+\left\|v_{k}-w_{k}\right\| \leq(1+\mu L)\left\|t_{k}-v_{k}\right\| .
$$

As follows from (3.2), (3.4) and $\mu L \in(0,1)$, we have

$$
\left\|w_{k}-q\right\|^{2} \leq\left\|t_{k}-q\right\|^{2}-\frac{1-\mu^{2} L^{2}}{(1+\mu L)^{2}}\left\|t_{k}-w_{k}\right\|^{2} .
$$

Let for any $q \in \Gamma$ and $T_{\sigma_{k}}:=\sigma_{k} V+\left(1-\sigma_{k}\right) U$. Now, by using (3.5), we estimate

$$
\begin{aligned}
\left\|u_{k+1}-q\right\|^{2}= & \left\|\left(1-\alpha_{k}\right) t_{k}+\alpha_{k} T_{\sigma_{k}} w_{k}-q\right\|^{2} \\
\leq & \left(1-\alpha_{k}\right)\left\|t_{k}-q\right\|^{2}+\alpha_{k}\left\|T_{\sigma_{n}} w_{k}-q\right\|^{2}-\alpha_{k}\left(1-\alpha_{k}\right)\left\|T_{\sigma_{k}} w_{k}-t_{k}\right\|^{2} \\
\leq & \left(1-\alpha_{k}\right)\left\|t_{k}-q\right\|^{2}+\alpha_{k}\left(\sigma_{k}\left\|V w_{k}-q\right\|^{2}+\left(1-\sigma_{k}\right)\left\|U w_{k}-q\right\|^{2}\right. \\
& \left.-\sigma_{k}\left(1-\sigma_{k}\right)\left\|V w_{k}-U w_{k}\right\|^{2}\right)-\alpha_{k}\left(1-\alpha_{k}\right)\left\|T_{\sigma_{k}} w_{k}-t_{k}\right\|^{2} \\
\leq & \left\|t_{k}-q\right\|^{2}-\alpha_{k} \sigma_{k}\left(1-\sigma_{k}\right)\left\|V w_{k}-U w_{k}\right\|^{2}-\frac{1-\mu^{2} L^{2}}{(1+\mu L)^{2}}\left\|t_{k}-v_{k}\right\|^{2} \\
& -\alpha_{k}\left(1-\alpha_{k}\right)\left\|T_{\sigma_{k}} w_{k}-t_{k}\right\|^{2} \\
\leq & \left\|t_{k}-q\right\|^{2}-\alpha_{k}\left(1-\alpha_{k}\right)\left\|T_{\sigma_{k}} w_{k}-t_{k}\right\|^{2} .
\end{aligned}
$$


Next, we estimate

$$
\begin{aligned}
\left\|t_{k}-q\right\|^{2}= & \left\|u_{k}+\eta_{k}\left(u_{k}-u_{k-1}\right)-q\right\|^{2} \\
= & \left(1+\eta_{k}\right)\left\|u_{k}-q\right\|^{2}-\eta_{k}\left\|u_{k-1}-q\right\|^{2} \\
& +\eta_{k}\left(1+\eta_{k}\right)\left\|u_{k}-u_{k-1}\right\|^{2} .
\end{aligned}
$$

From (3.7) and (3.8), we have

$$
\begin{aligned}
\left\|u_{k+1}-q\right\|^{2}-\left(1+\eta_{k}\right)\left\|u_{k}-q\right\|^{2}+\eta_{k}\left\|u_{k-1}-q\right\|^{2} \leq & -\alpha_{k}\left(1-\alpha_{k}\right)\left\|T_{\sigma_{k}} u_{k}-t_{k}\right\|^{2} \\
& +\eta_{k}\left(1+\eta_{k}\right)\left\|u_{k}-u_{k-1}\right\|^{2}
\end{aligned}
$$

Furthermore, from scheme (3.1), we have

$$
\begin{aligned}
\left\|T_{\sigma_{k}} w_{k}-t_{k}\right\|^{2}= & \left\|\frac{1}{\alpha_{k}}\left(u_{k+1}-u_{k}\right)+\frac{\eta_{k}}{\alpha_{k}}\left(u_{k-1}-u_{k}\right)\right\|^{2} \\
\geq & \frac{1}{\alpha_{k}^{2}}\left\|u_{k+1}-u_{k}\right\|^{2}+\frac{\eta_{k}^{2}}{\alpha_{k}^{2}}\left\|u_{k}-u_{k-1}\right\|^{2} \\
& +\frac{\eta_{k}}{\alpha_{k}^{2}}\left(-\rho_{k}\left\|u_{k+1}-u_{k}\right\|^{2}-\frac{1}{\rho_{k}}\left\|u_{k}-u_{k-1}\right\|^{2}\right),
\end{aligned}
$$

where $\rho_{k}:=\frac{1}{\eta_{k}+\delta \alpha_{k}}$. Thus, it follows from (3.9) and (3.10) that

$$
\begin{aligned}
& \left\|u_{k+1}-q\right\|^{2}-\left(1+\eta_{k}\right)\left\|u_{k}-q\right\|^{2}+\eta_{k}\left\|u_{k-1}-q\right\|^{2} \leq \frac{\left(1-\alpha_{k}\right)\left(\eta_{k} \rho_{k}-1\right)}{\alpha_{k}}\left\|u_{k+1}-u_{k}\right\|^{2} \\
& +\gamma_{k}\left\|u_{k}-u_{k-1}\right\|^{2}
\end{aligned}
$$

where

$$
\gamma_{k}:=\eta_{k}\left(1+\eta_{k}\right)+\eta_{k}\left(1-\alpha_{k}\right) \frac{\left(1-\eta_{k} \rho_{k}\right)}{\alpha_{k} \rho_{k}}>0,
$$

since $\eta_{k} \rho_{k}<1$ and $\alpha_{k} \in(0,1)$. It follows from $\delta=\frac{\left(1-\eta_{k} \rho_{k}\right)}{\alpha_{k} \rho_{k}}$ and (3.12) that

$$
\gamma_{k}:=\eta_{k}\left(1+\eta_{k}\right)+\eta_{k}\left(1-\alpha_{k}\right) \delta \leq \eta(1+\eta)+\eta \delta, \quad \forall k \geq 1
$$

Next, we define the sequences $\left\{\phi_{k}\right\}$ and $\left\{\psi_{k}\right\}$ by

$$
\phi_{k}:=\left\|x_{k}-q\right\|^{2}, \quad \psi_{k}:=\phi_{k}-\eta_{k} \phi_{k-1}+\gamma_{k}\left\|u_{k}-u_{k-1}\right\|^{2}, \quad \forall k \geq 1 .
$$

Now, using the monotonicity of $\left\{\eta_{k}\right\}$ and the fact that $\phi_{k} \geq 0$ for all $k \in \mathbb{N}$, we have

$$
\psi_{k+1}-\psi_{k} \leq \phi_{k+1}-\left(1+\eta_{k}\right) \phi_{k}+\eta_{k} \phi_{k-1}+\gamma_{k+1}\left\|u_{k+1}-u_{k}\right\|^{2}-\gamma_{k}\left\|u_{k}-u_{k-1}\right\|^{2} .
$$


Hence, it follows from (3.11) and (3.15) that

$$
\begin{aligned}
\psi_{k+1}-\psi_{k} & \leq \frac{\left(1-\alpha_{k}\right)\left(\eta_{k} \rho_{k}-1\right)}{\alpha_{k}}\left\|u_{k+1}-u_{k}\right\|^{2}+\gamma_{k+1}\left\|u_{k+1}-u_{k}\right\|^{2} \\
& =\left(\frac{\left(1-\alpha_{k}\right)\left(\eta_{k} \rho_{k}-1\right)}{\alpha_{k}}+\gamma_{k+1}\right)\left\|u_{k+1}-u_{k}\right\|^{2} .
\end{aligned}
$$

Now, we note that

$$
\frac{\left(1-\alpha_{k}\right)\left(\eta_{k} \rho_{k}-1\right)}{\alpha_{k}}+\gamma_{k+1} \leq-\sigma, \quad \forall k \geq 1
$$

see [9].

Therefore, it follows from (3.16) and (3.17) that

$$
\psi_{k+1}-\psi_{k} \leq-\sigma\left\|u_{k+1}-u_{k}\right\|^{2}
$$

Since $\eta_{1}=0$, it follows from (3.14) that $\psi_{1}=\phi_{1} \geq 0$ and hence (3.18) shows that $\left\{\psi_{k}\right\}$ is bounded. Furthermore, (3.14) and the boundedness of $\left\{\eta_{k}\right\}$ yield

$$
-\eta \phi_{k-1} \leq \phi_{k}-\eta \phi_{k-1} \leq \psi_{k} \leq \psi_{1}
$$

Thus, we obtain

$$
\phi_{k} \leq \eta^{k} \phi_{0}+\psi_{1} \sum_{j=1}^{k-1} \eta^{j} \leq \eta^{k} \phi_{0}+\frac{1}{1-\eta} \psi_{1} .
$$

Now, it follows from (3.18), (3.19), (3.20) and the boundedness of $\left\{\psi_{k}\right\}$ that

$$
\sigma \sum_{j=1}^{k}\left\|u_{j+1}-u_{j}\right\|^{2} \leq \psi_{1}-\psi_{k+1} \leq \psi_{1}+\eta \phi_{k} \leq \psi_{1}+\eta^{k} \phi_{0}+\frac{1}{1-\eta} \psi_{1},
$$

which implies that $\sum_{k=1}^{\infty}\left\|u_{k+1}-u_{k}\right\|^{2}<+\infty$.

Proof of (b). Since $\eta_{k} \rho_{k}<1$, it follows from (3.11), (3.13), $\sum_{k=1}^{\infty}\left\|u_{k+1}-u_{k}\right\|^{2}<+\infty$, and Lemma 2.2 that

$$
\lim _{k \rightarrow \infty}\left\|u_{k}-q\right\| \quad \text { exists and finite, }
$$

and hence $\left\{u_{k}\right\}$ is bounded. It follows furthermore from $\sum_{k=1}^{\infty}\left\|u_{k+1}-u_{k}\right\|^{2}<+\infty$ that

$$
\lim _{k \rightarrow \infty}\left\|u_{k+1}-u_{k}\right\|=0
$$

Next, by the definition of $t_{k}$ in (3.1) and $\eta_{k} \leq \eta, \forall k$, we have

$$
\left\|t_{k}-u_{k}\right\|=\eta_{k}\left\|u_{k}-u_{k-1}\right\| \leq \eta\left\|u_{k}-u_{k-1}\right\|,
$$


which implies that

$$
\lim _{k \rightarrow \infty}\left\|t_{k}-u_{k}\right\|=0
$$

and hence $\left\{t_{k}\right\}$ is bounded. Since

$$
\left\|t_{k}-u_{k+1}\right\| \leq\left\|t_{k}-u_{k}\right\|+\left\|u_{k}-u_{k+1}\right\|
$$

it follows from (3.23), (3.24) and (3.25) that

$$
\lim _{k \rightarrow \infty}\left\|t_{k}-u_{k+1}\right\|=0
$$

From (3.6) and (3.26), and $\left\{\alpha_{k}\right\} \subseteq(0,1),\left\{\sigma_{k}\right\} \subseteq[c, d], c, d \in(0,1)$, we have

$$
\begin{aligned}
\alpha_{k} \sigma_{k}\left(1-\sigma_{k}\right)\left\|V w_{k}-U w_{k}\right\|^{2} & =\left\|t_{k}-q\right\|^{2}-\left\|u_{k+1}-q\right\|^{2} \\
& \leq\left\|t_{k}-u_{k+1}\right\|\left(\left\|t_{k}-q\right\|+\left\|u_{k+1}-q\right\|\right) \\
& =\left\|t_{k}-u_{k+1}\right\| M_{1},
\end{aligned}
$$

where $M_{1}:=\sup _{k}\left\{\left\|t_{k}-q\right\|+\left\|u_{k+1}-q\right\|\right\}$. Hence, it follows

$$
\lim _{k \rightarrow \infty}\left\|V w_{k}-U w_{k}\right\|=0
$$

From (3.6) and (3.26), and $\mu L \in(0,1)$, we have

$$
\begin{aligned}
\frac{1-\mu^{2} L^{2}}{(1+\mu L)^{2}}\left\|t_{k}-w_{k}\right\|^{2} & \leq\left\|t_{k}-q\right\|^{2}-\left\|u_{k+1}-q\right\|^{2} \\
& =\left\|t_{k}-u_{k+1}\right\| M_{1},
\end{aligned}
$$

it follows that

$$
\lim _{k \rightarrow \infty}\left\|t_{k}-w_{k}\right\|=0
$$

It follows from (3.26) and (3.28) that

$$
\lim _{k \rightarrow \infty}\left\|t_{k}-u_{k+1}-\alpha_{k}\left(t_{k}-w_{k}\right)\right\|=0
$$

Furthermore, we have

$$
\begin{aligned}
& \alpha_{k}\left\|U w_{k}-w_{k}\right\| \leq\left\|u_{k+1}-t_{k}\right\|+\alpha_{k}\left\|t_{k}-w_{k}\right\|+\alpha_{k} \sigma_{k}\left\|U w_{k}-V w_{k}\right\|, \\
& \left\|U w_{k}-w_{k}\right\| \leq \frac{1}{\alpha_{k}}\left\|u_{k+1}-t_{k}\right\|+\left\|t_{k}-w_{k}\right\|+\sigma_{k}\left\|U w_{k}-V w_{k}\right\| .
\end{aligned}
$$

Since $\alpha_{k}>\alpha>0, \forall k$, it follows from (3.26), (3.27), (3.28) and (3.30) that

$$
\lim _{k \rightarrow \infty}\left\|U w_{k}-w_{k}\right\|=0
$$


From (3.27) and (3.31), we have

$$
\lim _{k \rightarrow \infty}\left\|V w_{k}-w_{k}\right\|=0
$$

Now, let $\bar{u}$ be a sequential weak cluster point of $\left\{u_{k}\right\}$, that is, there exists a subsequence $\left\{u_{k_{i}}\right\}$ of $\left\{u_{k}\right\}$ such that $\left\{u_{k_{i}}\right\}$ converges weakly to $\bar{u}$, say, in $\mathcal{H}$. Furthermore, (3.24) and (3.28) imply that $\left\{u_{k}\right\},\left\{t_{k}\right\}$ and $\left\{w_{k}\right\}$ all have the same asymptotic behavior and hence there exist subsequences $\left\{t_{k_{i}}\right\}$ of $\left\{t_{k}\right\}$ and $\left\{w_{k_{i}}\right\}$ of $\left\{w_{k}\right\}$ and such that $t_{k_{i}}$ and $w_{k_{i}}$ both converge weakly to $\bar{u}$. Now, Lemma 2.1, (3.31) and (3.32) imply that $\bar{u} \in \mathrm{F}(U)$ and $\bar{u} \in \mathrm{F}(V)$.

Next, we prove that $\bar{u} \in \Phi$. Since

$$
u_{k+1}-t_{k}=\alpha_{k}\left(w_{k}-t_{k}\right)+\alpha_{k}\left(\sigma_{k}\left(V w_{k}-w_{k}\right)+\left(1-\sigma_{k}\right)\left(U w_{k}-w_{k}\right)\right),
$$

and hence

$$
\frac{1}{\alpha_{k} \sigma_{k}}\left(t_{k}-u_{k+1}-\alpha_{k}\left(t_{k}-w_{k}\right)\right)=(I-V) w_{k}+\left(\frac{1-\sigma_{k}}{\sigma_{k}}\right)(I-U) w_{k} \text {, }
$$

and therefore for all $z \in \mathrm{F}(U)$ and by making use of the monotonicity of $I-V$, we have

$$
\begin{aligned}
\left\langle\frac{1}{\alpha_{k} \sigma_{k}}\left(t_{k}-u_{k+1}-\alpha_{k}\left(t_{k}-w_{k}\right)\right), w_{k}-z\right\rangle= & \left\langle(I-V) w_{k}-(I-V) z, w_{k}-z\right\rangle \\
& +\left\langle(I-V) z, w_{k}-z\right\rangle \\
& +\frac{1-\sigma_{k}}{\sigma_{k}}\left\langle w_{k}-U w_{k}, w_{k}-z\right\rangle \\
\geq & \left\langle(I-V) z, w_{k}-z\right\rangle \\
& +\frac{1-\sigma_{k}}{\sigma_{k}}\left\langle w_{k}-U w_{k}, w_{k}-z\right\rangle .
\end{aligned}
$$

Hence,

$$
\begin{gathered}
\left\langle\frac{1}{\alpha_{k_{i}} \sigma_{k_{i}}}\left(t_{k_{i}}-u_{k_{i}+1}-\alpha_{k_{i}}\left(t_{k_{i}}-w_{k_{i}}\right)\right), w_{k_{i}}-z\right\rangle \\
\geq\left\langle(I-V) z, w_{k_{i}}-z\right\rangle \\
\quad+\frac{1-\sigma_{k_{i}}}{\sigma_{k_{i}}}\left\langle w_{k_{i}}-U w_{k_{i}}, w_{k_{i}}-z\right\rangle .
\end{gathered}
$$

Using (3.29), (3.31), and the conditions on the parameters $\alpha_{k}$ and $\sigma_{k}$ in (3.36), we have

$$
\limsup _{i \rightarrow \infty}\left\langle z-V z, w_{k_{i}}-z\right\rangle \leq 0 \quad \forall z \in \mathrm{F}(U)
$$

Since $w_{k_{i}}$ converges weakly to $\bar{u}$, we get

$$
\langle(I-V) z, \bar{u}-z\rangle \leq 0, \quad \forall z \in \mathrm{F}(U)
$$

Since $\mathrm{F}(U)$ is convex, $\beta z+(1-\beta) \hat{u} \in \mathrm{F}(U)$ for $\beta \in(0,1)$ and hence

$$
\langle(I-V)(\beta z+(1-\beta) \bar{u}), \bar{u}-(\beta z+(1-\beta) \bar{u})\rangle
$$




$$
\begin{aligned}
& =\beta\langle(I-V)(\beta z+(1-\beta) \bar{u}), \bar{u}-z\rangle \\
& \leq 0, \quad \forall z \in \mathrm{F}(U),
\end{aligned}
$$

which implies

$$
\langle(I-V)(\beta z+(1-\beta) \bar{u}), \bar{u}-z\rangle \leq 0, \quad \forall z \in \mathrm{F}(U) .
$$

On taking the limit $\beta \rightarrow 0_{+}$, we have

$$
\langle(I-V) \bar{u}, \bar{u}-z\rangle \leq 0, \quad \forall z \in \mathrm{F}(U)
$$

which implies $\bar{u} \in \Phi$.

Now, we show that $\bar{u} \in \operatorname{Sol}(\operatorname{VI}(1.5))$. Since $\lim _{k \rightarrow \infty}\left\|v_{k}-t_{k}\right\|=0$ and $\lim _{k \rightarrow \infty}\left\|t_{k}-u_{k}\right\|=0$, there exist subsequences $\left\{t_{k_{i}}\right\}$ of $\left\{t_{k}\right\}$ and $\left\{v_{k_{i}}\right\}$ of $\left\{v_{k}\right\}$, respectively, such that $\left\{t_{k_{i}}\right\},\left\{v_{k_{i}}\right\}$ both converge weakly to $\bar{u}$. Let

$$
G v= \begin{cases}h v+N_{\mathcal{C}}(v), & \text { if } v \in \mathcal{C} ; \\ \emptyset, & \text { if } v \notin \mathcal{C},\end{cases}
$$

then the monotone mapping $G$ is maximal [32] and hence $0 \in G v$ if and only if $v \in$ $\operatorname{Sol}(\mathrm{VI}(1.5))$ [33]. Let $(v, w) \in \operatorname{graph}(G)$, then $w \in G v=h v+N_{\mathcal{C}}(v)$ and hence $w-h v \in N_{\mathcal{C}}(v)$, i.e., $\langle v-u, w-h v\rangle \geq 0$, for all $u \in \mathcal{C}$.

On the other hand, from $v_{k}=\mathcal{P}_{\mathcal{C}}(I-\mu h) t_{k}$ and $v \in \mathcal{C}$, we get

$$
\left\langle(I-\mu h) t_{k}-v_{k}, v_{k}-v\right\rangle \geq 0
$$

This implies that

$$
\left\langle v^{*}-v_{k}, \frac{v_{k}-t_{k}}{\mu}+h t_{k}\right\rangle \geq 0
$$

Since $\langle v-u, w-h v\rangle \geq 0$, for all $u \in \mathcal{C}$ and $v_{k_{i}} \in \mathcal{C}$, using the monotonicity of $h$, we have

$$
\begin{aligned}
\left\langle v-v_{k_{i}}, w\right\rangle & \geq\left\langle v-v_{k_{i}}, h v\right\rangle \\
& \geq\left\langle v-v_{k_{i}}, h v\right\rangle-\left\langle v-v_{k_{i}}, \frac{v_{k_{i}}-t_{k_{i}}}{\mu}+h t_{k_{i}}\right\rangle \\
& =\left\langle v-v_{k_{i}}, h v-h v_{k_{i}}\right\rangle+\left\langle v-v_{k_{i}}, h v_{k_{i}}-h t_{k_{i}}\right\rangle-\left\langle v-y_{k_{i}}, \frac{v_{k_{i}}-t_{k_{i}}}{\mu}\right\rangle \\
& \geq\left\langle v-v_{k_{i}}, h v_{k_{i}}-h t_{k_{i}}\right\rangle-\left\langle v-v_{k_{i}}, \frac{v_{k_{i}}-t_{k_{i}}}{\mu}\right\rangle
\end{aligned}
$$

Since $h$ is continuous, on taking the limit $i \rightarrow \infty$ we have $\langle v-\bar{u}, w\rangle \geq 0$. Since $G$ is maximal monotone, we have $\bar{u} \in G^{-1} 0$ and hence $\bar{u} \in \operatorname{Sol}(\operatorname{VI}(1.5))$ and thus $\bar{u} \in \Gamma$. 
Finally, it follows from (3.22) and Lemma 2.3 that the sequence $\left\{u_{k}\right\}$ converges weakly to $\bar{u} \in \Gamma$.

Remark 3.2 One can derive a number of schemes from scheme (3.1); some special cases are as follows:

(i) Setting $\eta_{k}=0, \forall k$ then scheme (3.1) reduces to extragradient scheme for solving VI(1.5) and H-FPP(1.1).

(ii) Setting $\sigma_{k}=0, \forall k$, and $V=I, U=I$ then scheme (3.1) reduces to scheme (1.8) for solving $\mathrm{VI}(1.5)$ and hence we recover Theorem 3.1 [25].

(iii) Setting $V=I, \sigma_{k}=0, U=J_{\lambda_{k}}^{B}:=\left(I+\lambda_{k} B\right)^{-1}$ (where $B: \mathcal{H} \rightarrow 2^{\mathcal{H}}$ is maximal monotone and $\lambda_{k} \in(0, \infty)$ ), and $\alpha_{k}=\alpha \forall k$, scheme (3.1) takes the following form:

$$
\left.\begin{array}{l}
t_{k}=u_{k}+\eta_{k}\left(u_{k}-u_{k-1}\right), \\
v_{k}=\mathcal{P}_{\mathcal{C}}\left(t_{k}-\mu h\left(t_{k}\right)\right), \\
w_{k}=\mathcal{P}_{\mathcal{C}}\left(t_{k}-\mu h\left(v_{k}\right)\right), \\
u_{k+1}=(1-\alpha) t_{k}+\alpha J_{\lambda_{k}}^{B} w_{k},
\end{array}\right\}
$$

which was considered with an additional error tolerance strategy in [34].

\section{Numerical example}

We discuss an example to illustrate Theorem 3.1.

Example 4.1 Let $\mathcal{H}=\mathbb{R}$. Let $\mathcal{C}=(-\infty,+\infty)$, the mappings $h: \mathcal{H} \rightarrow \mathcal{H}$ be defined by $h(u)=3 u-2, \forall u \in \mathcal{C}$; and $U, V: \mathcal{C} \rightarrow \mathcal{C}$ be defined by $U u=\frac{u+4}{7}, V u=\frac{u+6}{10}, \forall u \in \mathcal{C}$, respectively. Setting $\left\{\alpha_{k}\right\}=0.8,\left\{\eta_{k}\right\}=0.4$ and $\left\{\sigma_{k}\right\}=\left\{\frac{1}{1000}+\frac{0.9}{k^{2}}\right\}, \forall k \geq 1$. Then there are unique sequences $\left\{u_{k}\right\},\left\{v_{k}\right\}$ and $\left\{w_{k}\right\}$ obtained by scheme (3.1) converging to $\bar{u}=\frac{2}{3} \in \Gamma$.

Proof Since $h$ is Lipschitz continuous with $L=3$ and monotone and hence $\mu \in\left(0, \frac{1}{3}\right)$, we take $\mu=\frac{1}{4}$. Observe that the mappings $U, V$ are nonexpansive with $\mathrm{F}(U)=\left\{\frac{2}{3}\right\}, \mathrm{F}(V)=$ $\left\{\frac{2}{3}\right\}$, and hence $\Phi=\operatorname{Sol}(\mathrm{H}-\mathrm{FPP}(1.1))=\left\{\frac{2}{3}\right\}$. One can also obtain $\operatorname{Sol}(\mathrm{VI}(1.5))=\left\{\frac{2}{3}\right\}$. Hence, $\Gamma=\operatorname{Sol}(\mathrm{VI}(1.5)) \cap \Phi \cap \mathrm{F}(S)=\left\{\frac{2}{3}\right\} \neq \emptyset$. Furthermore, scheme (3.1) reduces to the following scheme: Given initial values $u_{0}, u_{1}$,

$$
\begin{aligned}
& t_{k}=u_{k}+\eta_{k}\left(u_{k}-u_{k-1}\right), \\
& v_{k}=\mathcal{P}_{\mathcal{C}}\left(t_{k}-\mu h\left(t_{k}\right)\right)= \begin{cases}0, & \text { if } u<0, \\
1, & \text { if } u>1, \\
\frac{1}{4} t_{k}+\frac{1}{2}, & \text { otherwise, }\end{cases} \\
& w_{k}=\mathcal{P}_{\mathcal{C}}\left(t_{k}-\mu h\left(v_{k}\right)\right)= \begin{cases}t_{k}+\frac{1}{2}, & \text { if } u<0, \\
t_{k}+\frac{1}{4}, & \text { if } u>1, \\
t_{k}-\frac{1}{4}\left(3 y_{k}-2\right), & \text { otherwise, }\end{cases} \\
& u_{k+1}=\left(1-\alpha_{k}\right) t_{k}+\alpha_{k}\left(\sigma_{k} \frac{w_{k}+6}{10}+\left(1-\sigma_{k}\right) \frac{w_{k}+7}{4}\right) .
\end{aligned}
$$

Finally, using MATLAB, we have Fig. 1 and Table 1 , which show that $\left\{u_{k}\right\},\left\{v_{k}\right\}$ and $\left\{w_{k}\right\}$ converge to $\bar{u}=\frac{2}{3}$ as $k \rightarrow+\infty$. 


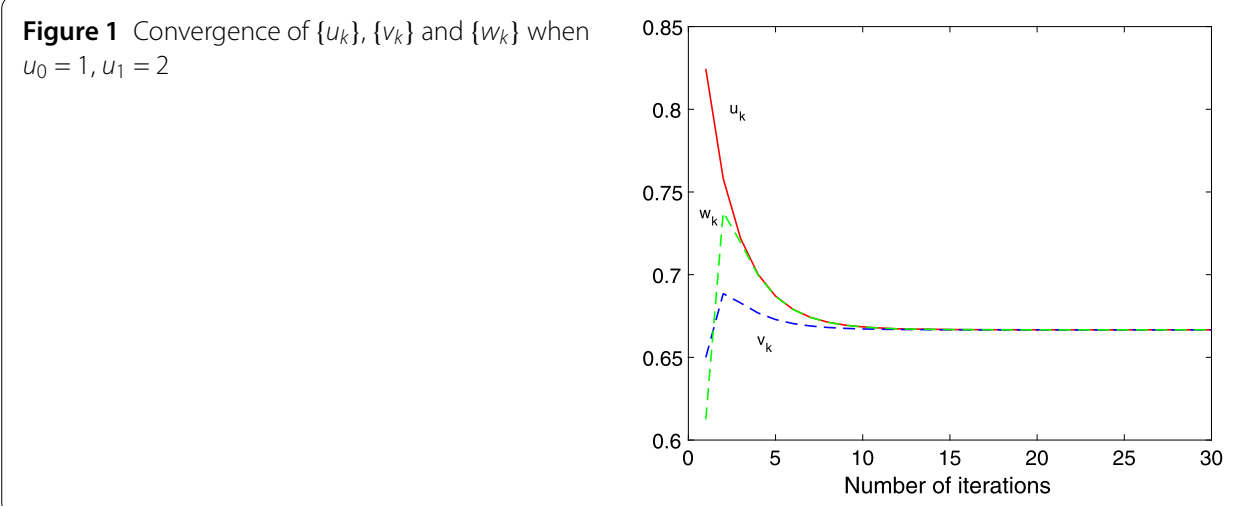

Table 1 Values of $u_{k}, v_{k}$ and $w_{k}$

\begin{tabular}{llll}
\hline No. of iterations & $u_{k}\left(u_{0}=1, u_{1}=2\right)$ & $v_{k}$ & $w_{k}$ \\
\hline 1 & 0.824408 & 0.650000 & 0.612500 \\
2 & 0.758075 & 0.688543 & 0.737764 \\
3 & 0.721779 & 0.682885 & 0.719378 \\
4 & 0.700135 & 0.676815 & 0.699649 \\
5 & 0.687021 & 0.672869 & 0.686825 \\
6 & 0.679051 & 0.670444 & 0.678942 \\
7 & 0.674203 & 0.668966 & 0.674138 \\
8 & 0.671253 & 0.668066 & 0.671214 \\
9 & 0.669458 & 0.667518 & 0.669434 \\
10 & 0.668366 & 0.667185 & 0.668351 \\
11 & 0.667701 & 0.666982 & 0.667692 \\
12 & 0.667296 & 0.666859 & 0.667291 \\
13 & 0.667050 & 0.666784 & 0.667047 \\
14 & 0.666900 & 0.666738 & 0.666898 \\
15 & 0.666809 & 0.666710 & 0.666807 \\
20 & 0.666679 & 0.666670 & 0.666678 \\
25 & 0.666668 & 0.666667 & 0.6666668 \\
29 & 0.666667 & 0.666667 & 0.666667 \\
30 & 0.666667 & 0.666667 & 0.6666667 \\
\hline
\end{tabular}

Concluding remark 4.1 In this paper, we considered a variational inequality problem (VI) and a hierarchical fixed point problem (H-FPP) in Hilbert space. We proposed an inertial version of Krasnoselski-Mann (KM)-type extragradient scheme (3.1) by combining the KM-type scheme (1.3) and an inertial version of the extragradient scheme (1.8) to approximate a common solution of H-FPP(1.1) and VI(1.5). Furthermore, we proved a weak convergence theorem for the proposed scheme (3.1). Finally, we discussed an example to illustrate Theorem 3.1.

\section{Acknowledgements}

This research was funded by the Deanship of Scientific Research at Princess Nourah bint Abdulrahman University through the Fast-track Research Funding Program. The authors sincerely thank the anonymous referees for their valuable suggestions and useful comments that have led to the present improved version of the original manuscript.

\section{Funding}

This research was funded by the Deanship of Scientific Research at Princess Nourah bint Abdulrahman University through the Fast-track Research Funding Program. 


\section{Competing interests}

The authors declare that they have no competing interests.

\section{Authors' contributions}

All authors contributed equally and study the final manuscript. All authors read and approved the final manuscript.

\section{Author details}

'Department of Mathematical Science, College of Science, Princess Nourah bint Abdulrahman University, P.O. Box 105862, Riyadh 11656, Saudi Arabia. ${ }^{2}$ Department of Mathematics, Jamia Millia Islamia, 110025 New Delhi, India.

${ }^{3}$ Deparment of Mathematics, Faculty of Science and Arts-Rabigh, King Abdulaziz University, P.O. Box 344, Rabigh 21911,

Saudi Arabia. ${ }^{4}$ Department of Mathematics, Aligarh Muslim University, 202002 Aligarh, India.

\section{Publisher's Note}

Springer Nature remains neutral with regard to jurisdictional claims in published maps and institutional affiliations.

\section{Received: 15 October 2020 Accepted: 3 February 2021 Published online: 22 February 2021}

\section{References}

1. Moudafi, A., Maige, P.-E.: Towards viscosity approximations of hierarchical fixed-point problems. Fixed Point Theory Appl. 2006, Article ID 95453 (2006)

2. Moudafi, A.: Krasnoselski-Mann iteration for hierarchical fixed-point problems. Inverse Probl. 23, 1635-1640 (2007)

3. Kazmi, K.R., Ali, R., Furkan, M.: Krasnoselski-Mann type iterative method for hierarchical fixed point problem and split mixed equilibrium problem. Numer. Algorithms 77, 289-308 (2018)

4. Kazmi, K.R., Ali, R., Furkan, M.: Hybrid iterative method for split monotone variational inclusion problem and hierarchical fixed point problem for a finite family of nonexpansive mappings. Numer. Algorithms 79, 499-527 (2018)

5. Dong, Q.L., Kazmi, K.R., Ali, R., Li, X.H.: Inertial Krasnoseski-Mann type hybrid algorithms for solving hierarchical fixed point problems. J. Fixed Point Theory Appl. 21, 57 (2019)

6. Moudafi, A., Maige, P.-E.: Strong convergence of an iterative method for hierarchical fixed-point problems. Pac. J. Optim. 3, 529-538 (2007)

7. Yao, Y., Liou, Y.C.: Weak and strong convergence of Krasnoselski-Mann iteration for hierarchical fixed-point problems. Inverse Probl. 24, 501-508 (2008)

8. Maingé, P.E.: Convergence theorem for inertial KM-type algorithms. J. Comput. Appl. Math. 219, 223-236 (2008)

9. Bot, R.I., Csetnek, E.R., Hendrich, C.: Inertial Douglas-Rachford splitting for monotone inclusion problems. Appl. Math. Comput. 256, 472-487 (2015)

10. Tan, B., Li, S.: Strong convergence of inertial Mann algorithms for solving hierarchical fixed point problems. J. Nonlinear Var. Anal. 4, 337-355 (2020)

11. Tan, B., Cho, S.Y.: An inertial Mann-like algorithm for fixed points of nonexpansive mappings in Hilbert spaces. J. Appl. Numer. Optim. 2, 335-351 (2020)

12. Tian, M., Xu, G.: Inertial modified Tseng's extragradient algorithms for solving monotone variational inequalities and fixed point problems. J. Nonlinear Funct. Anal. 2020, Article ID 35 (2020)

13. Zhang, L., Zhao, H., Lv, Y.: A modified inertial projection and contraction algorithms for quasi-variational inequalities Appl. Set-Valued Anal. Optim. 1, 63-76 (2019)

14. Hartman, P., Stampacchia, G.: On some non-linear elliptic differential-functional equation. Acta Math. 115, 271-310 (1966)

15. Korpelevich, G.M.: The extragradient method for finding saddle points and other problems. Matecon 12, 747-756 (1976)

16. Ceng, L.C., Petrusel, A., Yao, J.C., Yao, Y.: Hybrid viscosity extragradient method for systems of variational inequalities, fixed points of nonexpansive mappings, zero points of accretive operators in Banach spaces. Fixed Point Theory 19, 487-502 (2018)

17. Ceng, L.C., Petrusel, A., Yao, J.C., Yao, Y.: Systems of variational inequalities with hierarchical variational inequality constraints for Lipschitzian pseudocontractions. Fixed Point Theory 20, 113-133 (2019)

18. Censor, Y., Gibali, A., Reich, S.: The subgradient extragradient method for solving variational inequalities in Hilbert space. J. Optim. Theory Appl. 148, 318-335 (2011)

19. He, B.S.: A new method for a class of variational inequalities. Math. Program. 66, 137-144 (1994)

20. Solodov, M.V., Tseng, P.: Modified projection-type methods for monotone variational inequalities. SIAM J. Control Optim. 34, 1814-1830 (1996)

21. Yao, Y., Liou, Y.C., Yao, J.C.: Iterative algorithms for the split variational inequality and fixed point problems under nonlinear transformations. J. Nonlinear Sci. Appl. 10, 843-854 (2017)

22. Yao, Y., Postolache, M., Yao, J.C.: Iterative algorithms for the generalized variational inequalities. UPB Sci. Bull., Ser. A 81, 3-16 (2019)

23. Yao, Y., Postolache, M., Yao, J.C.: Iterative algorithms for pseudomonotone variational inequalities and fixed point problems of pseudocontractive operators. Mathematics 7, 1189 (2019)

24. Yao, Y., Postolache, M., Yao, J.C.: Strong convergence of an extragradient algorithm for variational inequality and fixed point problems. UPB Sci. Bull., Ser. A 82(1), 3-12 (2020)

25. Dong, Q.L., Lu, Y.Y., Yang, J.: The extragradient algorithm with inertial effects for solving the variational inequality. Optimization 65(2), 2217-2226 (2016)

26. Combettes, P.L.: Quasi-Fejerian analysis of some optimization algorithms. In: Butnariu, D., Censor, Y., Reich, S. (eds.) Inherently Parallel Algorithms in Feasbility and Optimization and Their Applications. Elsevier, New York (2001)

27. Yang, Q., Zhao, J.: Generalized km theorem and their applications. Inverse Probl. 22, 833-844 (2006)

28. Byrne, C.: A unified treatment of some iterative algorithms in signal processing and image reconstruction. Inverse Probl. 20, 103-120 (2004) 
29. Geobel, K., Kirk, W.A.: Topics in Metric Fixed Point Theory. Cambridge Studies in Advanced Mathematics. Cambridge University Press, Cambridge (1990)

30. Alvarez, F.: Weak convergence of a relaxed and an inertial hybrid projection-proximal point algorithm for maximal monotone operators in Hilbert space. SIAM J. Optim. 14, 773-782 (2004)

31. Opial, Z: Weak convergence of the sequence of successive approximations for nonexpansive mappings. Bull. Am. Math. Soc. 73(4), 595-597 (1967)

32. Brẽzis, H.: Operateurs maximaux monotones et semi-groupes de contractions dans les espaces de Hilbert. In: Mathematical Studies, vol. 5, pp. 759-775. North-Holland, Amsterdam (1973)

33. Nadezhkina, N., Takahashi, W.: Weak convergence theorem by an extragradient method for nonexpansive mappings and monotone mappings. J. Optim. Theory Appl. 128, 191-201 (2006)

34. Alvarez, F:: On the minimizing property of a second order dissipative system in Hilbert spaces. SIAM J. Control Optim. 38(4), 1102-1119 (2000)

Submit your manuscript to a SpringerOpen ${ }^{\circ}$ journal and benefit from:

- Convenient online submission

Rigorous peer review

Open access: articles freely available online

- High visibility within the field

- Retaining the copyright to your article

Submit your next manuscript at $\boldsymbol{\triangleright}$ springeropen.com 